\title{
Dynamic behaviour of unstiffened stainless steel profiled barrier blast walls
}

\author{
Mohammad H. Hedayati ${ }^{1}$, Srinivas Sriramula ${ }^{2}$ and Richard D. Neilson ${ }^{3}$ \\ ${ }^{1} \mathrm{PhD}$ Student, School of Engineering, University of Aberdeen, UK; e-mail: \\ mohammad.hedayati@abdn.ac.uk \\ ${ }^{2}$ Lloyd's Register Foundation (LRF) Centre for Safety and Reliability Engineering, School of \\ Engineering, University of Aberdeen, UK; e-mail: s.sriramula@abdn.ac.uk \\ ${ }^{3}$ School of Engineering, University of Aberdeen, UK; e-mail: r.d.neilson@abdn.ac.uk
}

\begin{abstract}
:
Performing optimum design, reliable assessment or suitable verification for stainless steel profiled barrier blast wall structures requires dealing with various challenges, stemming from the associated uncertainties in material properties, fabrication, installation, and more importantly variations in the blast load characteristics. In the analysis, assessment, and design of these blast walls, one of the key areas to be appreciated and understood is the dynamic response of these structures. This paper presents a methodology developed for identifying the predominant structural behaviour and characteristics of profiled barrier blast wall structures, using a probabilistic approach. Twenty parametric base models are developed using Ansys and by implementing a Latin Hypercube Sampling (LHS) approach, the section properties of the models are represented in terms of probability distributions. A number of models are generated stochastically and modal analyses performed to identify the dynamic sensitivity of these models. The corresponding response classification of these structures is evaluated from the load duration and natural periods of the structures. The results of the study confirm that structural response, for the wide range of profiled blast walls analysed, is mainly quasi-static or static, as opposed to dynamic. In fact, dynamic effects are negligible for unstiffened
\end{abstract}


profiled barrier blast walls and structural responses in most cases can be estimated on a static or quasi-static basis. This conclusion would help a competent design engineer to consider a proper dynamic load factor at an early stage of the design, without involving complex advanced nonlinear dynamic analyses.

Keywords: Stainless steel blast walls; Unstiffened profiled barriers; Modal analysis; Probabilistic analysis; Ansys; Latin Hypercube Sampling.

\section{Abbreviations}

ABS: American Bureau of Shipping

APDL: ANSYS Parametric Design Language

API: American Petroleum Institute

ASD: Allowable Stress Design

CDF: Cumulative Density Function

DAF: Dynamic Amplification Factor

DLF: Dynamic Load Factor

DNV: Det Norske Veritas

FABIG: Fire and Blast Information Group

FEA: Finite Element Analysis

LHS: Latin Hypercube Sampling

LRFD: Load and Resistance Factor Design

MCS: Monte Carlo Simulation

MDOF: Multi Degree of Freedom

NLFEA: Nonlinear Finite Element Analysis

SDOF: Single Degree of Freedom

TN: Technical Note 


\section{Nomenclature}

$n \times k$ : matrix of input variables

\section{L1, L2, L3, S, L and H: the section geometry of blast wall}

$P_{0}:$ peak dynamic pressure

$t$ : time

$T$ : Natural period of structure

$t_{d}:$ duration of applied load

Tw: thickness of blast wall

$\Theta$ : section angle

\section{Introduction and Background}

Compared to other possible ways of protection against explosions, blast walls have lower cost/strength ratio and can be installed very quickly (Haifu and Xueguang 2009). Blast wall structures can be formed of stiffened or unstiffened panels; however, stainless steel profiled walls have increasingly been used in the offshore industry because of their excellent energy absorption and temperature dependent properties (Brewerton and FABIG TN5. 1999; Louca and Boh 2004).

In general, when considering the deterministic response of profiled barrier structures, two approaches are usually recommended for the design: the traditional Single Degree of Freedom (SDOF) approach or the more sophisticated Multi Degree of Freedom (MDOF) approach. The simplified SDOF approach is widely used in the offshore industry for predicting the dynamic structural response by implementing the Biggs method (Biggs 1964). This is a simple approach which idealizes the actual structure as a spring/mass model and is thus very useful in routine design procedures to obtain accurate results for relatively simple 
structures with limited ductility (Louca and Boh 2004). The SDOF approach is a useful technique for conceptual or basic design of the profiled barrier structures under explosion loadings, whereas, the MDOF method, which is typically based on a Finite Element Analysis (FEA) approach, provides a detailed analysis of the blast wall and is more accurate when compared to the SDOF approach, but is computationally very intensive and, as a result, more expensive. However, with recent developments in computing technology, performing FEA is easier and faster than it was in the past. There have also been some preliminary studies to verify SDOF results against MDOF results (Liang et al. 2007). Nonlinear FEA (NLFEA) can be used to overcome the limitations of normally implemented analytical methods which have mostly been developed to study elastic response or some specific plastic response and do not allow for large deformations and unstable responses. In addition, the use of NLFEA can prepare the way for a more rigorous performance-based blast resistant design or assessment, since the current principles as set out in the design guides cannot be fully adequate.(Boh et al. 2007). One of the main benefits of using the NLFEA approach is to capture local effects and to take into account buckling failure modes in the analyses and assessments. A study was carried out by Sun and Spencer (2005) on the buckling strength assessment of corrugated panels. They noticed that the most efficient way to enhance the buckling strength of corrugated panels is to increase the trough depth. The results also confirm that the recommended approach and formulas developed within the ABS guidelines (ABS. 2005; ABS. 2004) have suitable conservatisms for the buckling strength assessment and the FEA method with the correct boundary conditions gives more realistic results than the guidelines. Another study was performed by Czujko and Paik (2015), on the structural damage and robustness of blast walls designed for topside platforms. They highlighted that the use of precise analysis methods, such as NLFEA should allow optimisation of the design and to have proper required strengthening for the blast walls. They supported their study by 
implementing detailed non-linear finite element (FE) analysis, including static and dynamic assessments.

As another example, a further study was conducted by Sohn et al. (2016), investigating the effects of stiffeners to reduce the probability of having local failures. They concluded that a flat stiffener can be located in the buckling regions to improve the buckling strength of blast walls. There have been various studies performed to investigate the local failures, including buckling, of stainless steel profiled sections. Many of these studies have also confirmed that using the developed equations in guidelines can lead to conservative or unrealistic strength and stability predictions (e.g., (Hancock et al. 1990)) and therefore, a realistic finite element analysis would be advantageous and is recommended.

Although it is found that using a finite element approach has various benefits to perform probabilistic and reliability analyses, care should be taken while using this approach. One useful approach would be to advance the developments step by step, in such a way that each stage can be checked properly or compared with other stages if it is required. Therefore, with regard to this, a preliminary static finite element probabilistic approach on profiled barrier blast walls was undertaken by two of the authors (Hedyati and Sriramula 2012). The study was then extended by considering the dynamic effects and nonlinearities in geometric and material properties (Hedayati et al. 2013). It was noticed that considering the dynamic and non-linearity effects, the correlation sensitivity results are not similar at different time steps in the blast simulation. This study resulted in further investigations on using linear dynamic analysis, without implementing any non-linearity effects, to review and understand the linear dynamic behaviour of these structures under explosion loading (Hedayati et al. 2014). The sensitivity results of this study confirmed that the maximum response is not sensitive to duration of loading, indicating that the response of the structure is less dynamic. In the previous studies associated with this research, it was found that performing linear, non- 
linear, static, and dynamic analyses are crucial to assess the relative importance of the dynamic and non-linearity effects while implementing reliability approach. Accordingly, it was observed that the effects of nonlinearities are crucial to be considered in the assessments (Hedayati et al. 2015). It was also noticed that there is no profound difference between probabilistic responses for static and dynamic analyses, suggesting that a better realisation of employing an appropriate dynamic load factor (DLF) is very significant. This paper therefore presents the investigations carried out using the proposed framework, on the dynamic behaviour sensitivity of typical profiled barrier structures, implementing a probabilistic approach.

Initially, a parametric finite element (FE) model was developed using the ANSYS Parametric Design Language (APDL). Twenty FE base models were developed based on previous research by the authors. Then, by employing the verified base models, $40000 \mathrm{FE}$ representations were generated after introducing random input and output variables. As the dynamic characteristic (i.e. natural period) of the structure is associated with the mass and stiffness, by defining the uncertainties in different section properties as random variables, it is possible to implement efficiently simulation strategies for assessing the dynamic structural performance. The response parameters can be obtained by linking the simulated values with the finite element models. In the present study, a Latin Hypercube Sampling (LHS) approach was used to study the statistically significant results to identify the dynamic characteristics and performance of the blast walls.

\section{Design Approach for Profiled Blast Wall Structures}

The Design Guide for stainless steel blast walls, known as the Technical Note 5 (TN5) (Brewerton and FABIG TN5. 1999) prepared by the Fire and Blast Information Group (FABIG) and API Recommended Practice 2FB (API 2FB April 2006) are the two most 
commonly used technical industrial guidelines for the design of profiled blast walls, based on the SDOF method. One of the key areas that should be considered in the design and assessments of the profiled barrier blast walls is to apply a proper dynamic load factor (DLF), which represents the dynamic characteristic of the structure. Therefore, understanding the dynamic behaviour of the structure is vital and could help the process of analysis, assessment and design for profiled barrier blast walls.

In the present study, twenty profiled wall sections that satisfy the geometric limits, required to allow the wall to be deemed an appropriate structural element in accordance with the design guidance from TN5, are considered. The stainless steel sections considered are assumed to have a nominal Young's modulus of 200 GPa, Poisson's ratio of 0.3 and material density of $7,850 \mathrm{~kg} / \mathrm{m}^{3}$. Figure 1 gives an overview of the wall and the geometry of the profiled barrier section.
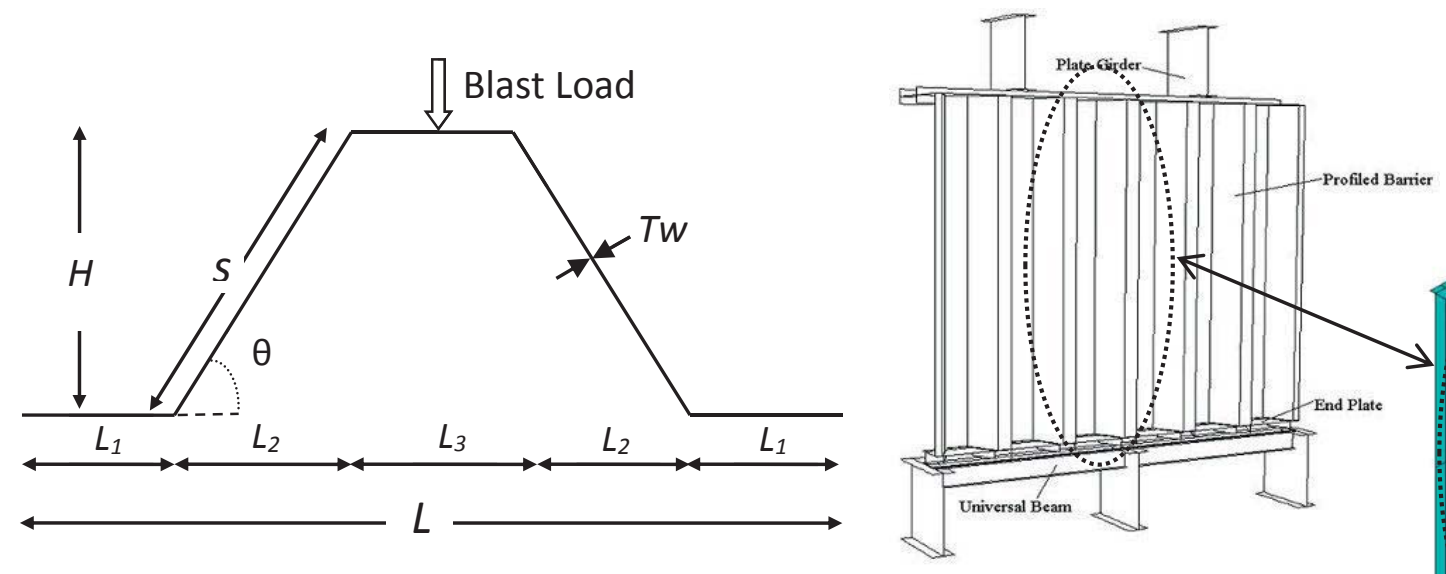

Figure 1. Overview of blast wall and the geometry of section 


\section{Probabilistic Approach}

\subsection{Model Uncertainties and Latin Hypercube Sampling}

One dominant aspect in the probabilistic assessment of profiled barrier blast walls is to identify uncertainties, stemming from various sources, and then implement them accurately in the associated analyses. In this study, to have a wide range of random modal analysis models, geometric properties are introduced as the uncertainties and are considered in the probabilistic analyses by modelling the properties as random variables represented by probability distributions. Probabilistic analysis results can be sensitive to the tail of the probability distribution and therefore, an appropriate approach/method to select the proper distribution type is necessary (Det Norske Veritas 1992). In this study, for all of the random variables, except for the profiled barrier thickness, Tw, which uses the normal or Gaussian distribution is assumed, for demonstrative purposes.

Probabilistic analysis based on the Monte Carlo Simulation (MCS) is widely used because of the ease of implementation, and the ability to handle complex engineering problems. However, when probabilities of occurrence are very small, the computational effort increases significantly. In such cases, it is possible to reduce the required number of simulations by using an appropriate variation reduction scheme such as importance sampling, Latin Hypercube Sampling (LHS) or directional simulation (Choi et al. 2006).

In the present study, an LHS scheme is used. A major advantage of LHS is that it avoids repeated sample reliability evaluations (ANSYS 2012; Reh et al. 2006), thus drastically reducing the number of simulations. LHS also considers the tails of the distributions more accurately. This is very important for most structural engineering applications where extreme values are important. 
The LHS technique was first introduced by McKay et al. (1979). Later on, further developments were explained by other researchers, for example by Iman et al. (1981). A typical Latin hypercube sampling selects $n$ different values from each of the $k$ variables $X_{1} \ldots$ $X_{k}$ as per the following routine (Wyss and Jorgensen 1998):

- The range of each variable is divided into $n$ non-overlapping intervals on the basis of equal probability.

- One value from each interval is selected at random with respect to the probability density in the interval.

- The $n$ values thus obtained for $X_{1}$ are paired in a random manner (equally likely combinations) with the $n$ values of $X_{2}$. These $n$ pairs are combined in a random manner with the $n$ values of $X_{3}$ to form $n$ triplets, and so on, until $n k$-tuplets are formed; these $n k$-tuplets are the same as the $n k$-dimensional input vectors.

It is convenient to think of this Latin hypercube sample (or any random sample of size $n$ ) as forming an $(n \times k)$ matrix of inputs where the $i^{\text {th }}$ row contains specific values of each of the $k$ input variables to be used on the $i^{\text {th }}$ run of the computer model. A more detailed description of LHS and the associated computer codes and manuals are given by Wyss and Jorgensen (1998). Figure 2 presents a two dimensional Latin hypercube sampling scheme.

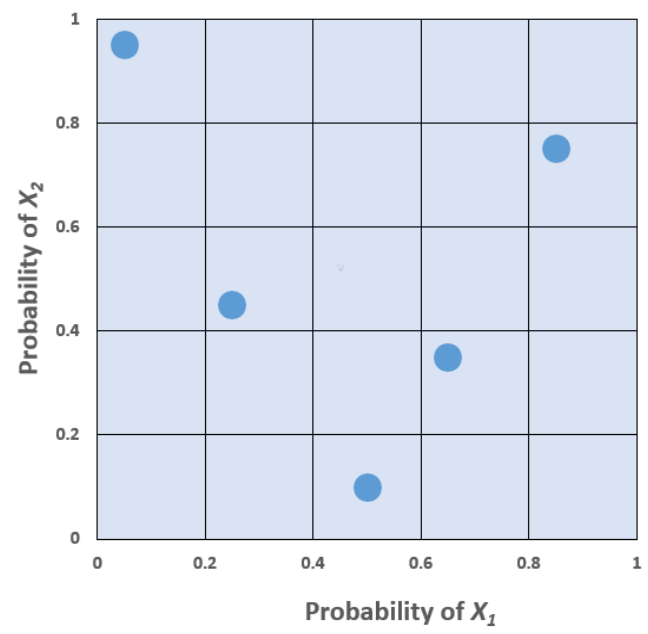

Figure 2. Two dimensional Latin hypercube sampling scheme 
This scheme has been further developed for different purposes by several researchers, e.g., Helton and Davis (2003) and Olsson et al. (2003).

\subsection{Analysis Models}

To investigate the dynamic behaviour of a structure, modal analysis is one of the key analysis types to be carried out to identify the sensitivity of stiffness and mass properties of the structure to dynamic loading properties, including duration of the loading. To expedite the probabilistic assessment, a beam model, as oppose to a shell model or SDOF system, was selected. The finite element beam modelling approach has sufficient accuracy for this study as well as limiting the number of elements which results in speeding up the process for the probabilistic analyses. Twenty finite element beam base-models of blast walls were employed in this study: ten base models were selected from the previous studies carried out by other researchers and another ten base models were developed by authors considering the FABIG guidelines (i.e. the appropriate dimensions and section properties which satisfy the guideline). It should be noted that this part of study deals purely with modal analyses, using the verified beam models and performing probabilistic analyses to investigate dynamic performance of unstiffened profiled barrier blast walls.

Based on FABIG TN5 (Brewerton and FABIG TN5. 1999), the section angle $(\theta)$, shown in Figure 1, should be limited to between 45 and 90 degrees to ensure good performance of the section and to prevent local failure. However, this limitation can be reviewed and implemented case by case by competent engineers, as considered differently by Schleyer et al. (2003) and Langdon and Schleyer (2006). Table 1 presents the section properties such as thickness and dimensions, span, angle $(\theta)$ and the natural period of the structure associated with the presented properties. Figure 3 gives an overview of the relative section sizes for the three first models (i.e. "Model1", "Model2", and "Model3") shown in the Table 1. 
The twenty deterministic base-line models have six parameters that are regarded as random input variables. These variables along with their assumed distribution models and parameters are given in Table 2. The random input variables are assumed to be statistically independent. Typical probability density and cumulative distribution functions of the span and section height are shown in Figure 4. For each model, 2000 runs were randomly generated by introducing the random input variables with the associated parametric variables for probabilistic analyses.

The details of the upper and lower connections of the blast walls have not been considered in the assessments, for two main reasons. Firstly, for the model verification, developing connections using springs for the SDOF modelling or using beam elements for the beam modelling was not practical. In fact, there would be some other uncertainties added to the models, which are hard, or in some cases, impossible to justify. Furthermore, for the second main part of the study, which is associated with probabilistic analyses, 40000 connections would need to be developed probabilistically resulting in more complexity as each individual connection would need to be validated.

In this study, according to the recommendation from the Technical Note 5 (Brewerton and FABIG TN5. 1999) and on consideration of the effective span with no upper and lower connection details, pinned-end fixity for the two ends of structure was implemented for the upper and lower boundary conditions. In addition, apart from the horizontal displacement along the wall, all out-of-plane and in-plane rotations and displacement along the supporting edge were constrained. 
Table 1. Mean values of geometry properties for selected and developed models

\begin{tabular}{|c|c|c|c|c|c|c|c|c|c|}
\hline $\begin{array}{l}\text { Model } \\
\text { Name }\end{array}$ & $\begin{array}{c}T w \\
(\mathrm{~mm})\end{array}$ & $\begin{array}{c}L 1 \\
(\mathrm{~mm})\end{array}$ & $\begin{array}{c}\mathrm{L2} \\
(\mathrm{mm})\end{array}$ & $\begin{array}{c}\text { L3 } \\
(\mathrm{mm})\end{array}$ & $\begin{array}{c}H \\
(\mathrm{~mm})\end{array}$ & $\begin{array}{l}\text { Span } \\
(\mathrm{mm})\end{array}$ & $\begin{array}{c}\theta \\
\text { (Deg.) }\end{array}$ & $\begin{array}{c}\text { Natural } \\
\text { Period }\end{array}$ & Reference \\
\hline & & & & & & & & & (Louca and Boh \\
\hline \multirow{2}{*}{ Model1 } & 11 & 200 & 320 & 240 & 554 & 6000 & 60 & 0.025 & 2004) \\
\hline & & & & & & & & & (Louca and Boh \\
\hline \multirow{2}{*}{ Model2 } & 9 & 160 & 160 & 160 & 200 & 4000 & 51 & 0.029 & 2004) \\
\hline & & & & & & & & & (Louca and Boh \\
\hline \multirow[t]{2}{*}{ Model3 } & 2.5 & 62.5 & 40 & 45 & 45 & 2322 & 48 & 0.053 & 2004) \\
\hline & & & & & & & & & (Schleyer and \\
\hline \multirow[t]{3}{*}{ Model4 } & 2 & 17.5 & 50 & 85 & 40.5 & 915 & 39 & 0.010 & Langdon 2003) \\
\hline & & & & & & & & & (RW.ERROR - \\
\hline & & & & & & & & & Unable to find \\
\hline Model5 & 8 & 70 & 200 & 340 & 162 & 3640 & 39 & 0.031 & reference:58) \\
\hline Model6 & 9 & 70 & 128 & 120 & 160 & 4000 & 51.3 & 0.037 & (Faruqi et al. 2010) \\
\hline Model7 & 10 & 80 & 126 & 140 & 180 & 4000 & 55 & 0.032 & (Faruqi et al. 2010) \\
\hline \multirow[t]{2}{*}{ Model8 } & 11 & 160 & 46 & 160 & 260 & 4000 & 80 & 0.022 & (Faruqi et al. 2010) \\
\hline & & & & & & & & & (Brewerton and \\
\hline Model9 & 5 & 200 & 200 & 400 & 300 & 4850 & 56.3 & 0.030 & FABIG TN5. 1999) \\
\hline Model10 & 2.5 & 90 & 119 & 180 & 150 & 3000 & 51.6 & 0.027 & $\begin{array}{l}\text { (Wijaya and Kim } \\
\text { 2011) }\end{array}$ \\
\hline Model11 & 11 & 100 & 90 & 300 & 520 & 5500 & 80.2 & 0.023 & Authors \\
\hline Model12 & 10 & 100 & 120 & 260 & 360 & 5000 & 71.6 & 0.026 & Authors \\
\hline Model13 & 9 & 80 & 140 & 240 & 330 & 4800 & 67.0 & 0.027 & Authors \\
\hline Model14 & 8 & 80 & 135 & 200 & 280 & 4600 & 64.3 & 0.029 & Authors \\
\hline Model15 & 7 & 70 & 120 & 180 & 220 & 4000 & 61.4 & 0.028 & Authors \\
\hline Model16 & 6 & 70 & 110 & 170 & 180 & 3600 & 58.6 & 0.028 & Authors \\
\hline Model17 & 5 & 70 & 100 & 180 & 170 & 3200 & 59.5 & 0.024 & Authors \\
\hline Model18 & 4 & 60 & 90 & 150 & 145 & 2800 & 58.2 & 0.022 & Authors \\
\hline Model19 & 3 & 50 & 80 & 120 & 110 & 2500 & 54.0 & 0.025 & Authors \\
\hline Model20 & 2 & 20 & 50 & 70 & 60 & 2200 & 50.2 & 0.040 & Authors \\
\hline
\end{tabular}




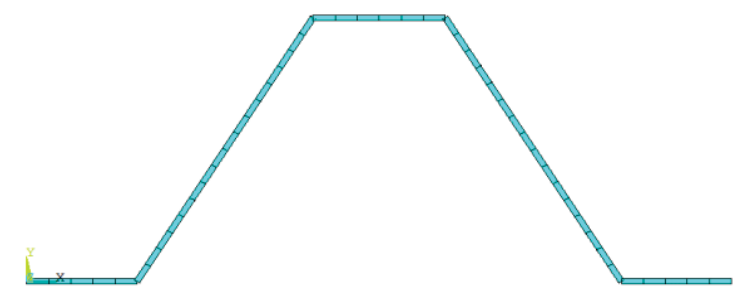

Model1

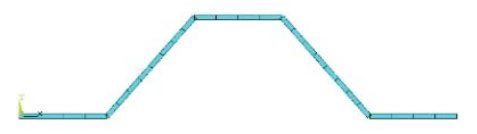

Model2

Model3

Figure 3. An overview of scaled-section sizes of the model 1, 2\&3

Table 2. Parametric Variables for Probabilistic Analysis

\begin{tabular}{lllllll}
\hline $\begin{array}{l}\text { Random } \\
\text { variable }\end{array}$ & $\begin{array}{l}\text { Height, } \\
H(\mathrm{~mm})\end{array}$ & $\begin{array}{l}\text { Thickness, } \\
T w(\mathrm{~mm})\end{array}$ & L1 & L2 & L3 & Span \\
\hline $\begin{array}{l}\text { Coefficient } \\
\text { of variation }\end{array}$ & 0.1 & $\sim 0.1$ & 0.15 & 0.15 & 0.15 & 0.15 \\
$\begin{array}{l}\text { Probability } \\
\text { distribution }\end{array}$ & Gaussian & Uniform & Gaussian & Gaussian & Gaussian & Gaussian \\
\hline
\end{tabular}
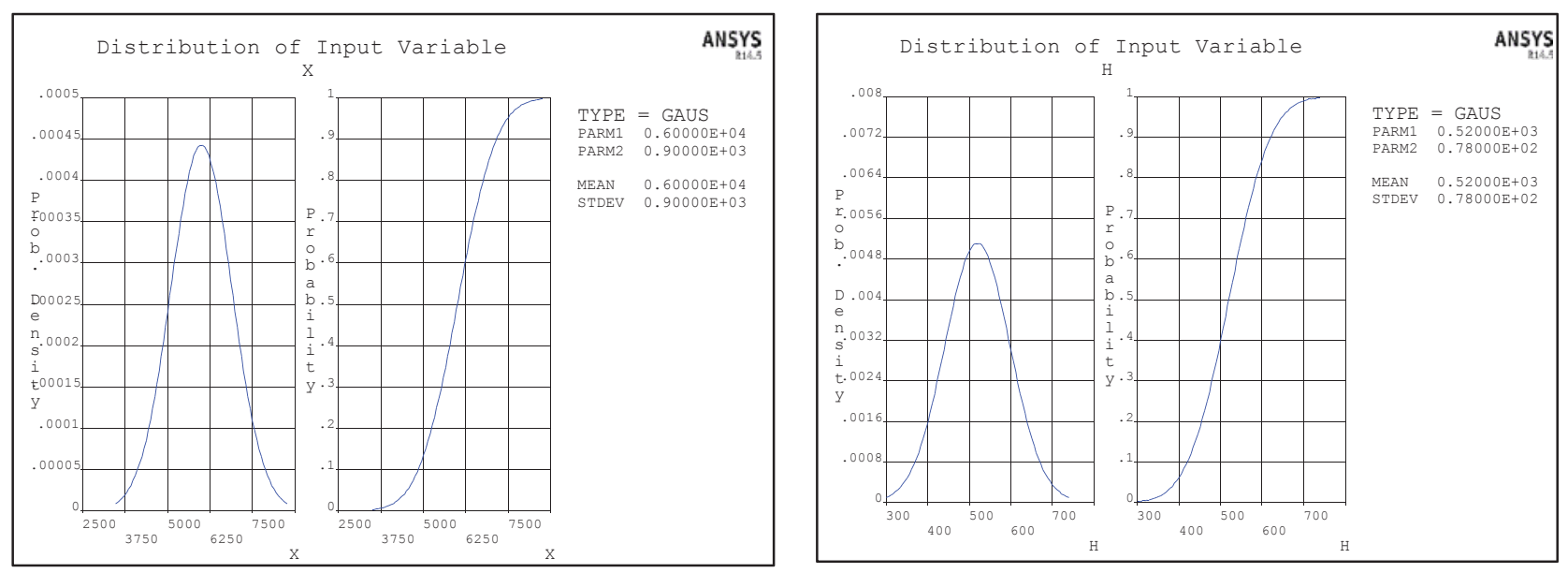

Figure 4. Distribution of Span (mm) (Left) and H (mm) (Right) 


\subsection{Modal validation}

Before performing modal analyses and implementing a reliability or probabilistic approach, the models should be verified to ensure model accuracy. There are various types of verification methods including analytical approaches, numerical methods and physical tests. For this study, the numerical finite element method was selected to validate the developed finite element models. To verify each of the twenty base-models, three analysis modelling systems comprising SDOF, Beam and Shell models were developed initially for each using the geometry and section properties presented by Table 1. For the dynamic transient analyses, a triangular load pulse with a peak dynamic pressure $\left(P_{0}\right)$ of 2.0 bar is used. The total time duration $\left(t_{d}\right)$ for this load pulse is 0.15 seconds. Figure 5 gives a view of the displacement results for the three models and confirms good agreement between the results. As can be seen from Figure 5, although there is a small shift between the results after the time of loading (i.e. $t_{d}>0.15 \mathrm{sec}$ ), the trend and overall behaviour of the structure is very similar with little difference in the maximum response. The response is elastic-plastic (i.e., not fully plastic), and as the boundary conditions for the end connections are pinned, a full plastic condition would cause collapse and consequently a full response would not be achievable. Comparing the results of these three modelling approach, it can be concluded that the developed numerical finite element models presented in this study have enough accuracy for the analysis and assessment and the SDOF or beam models can be used with little loss of accuracy compared to the full shell model. The same approach was implemented to validate all the twenty base models presented by Table 1 . 


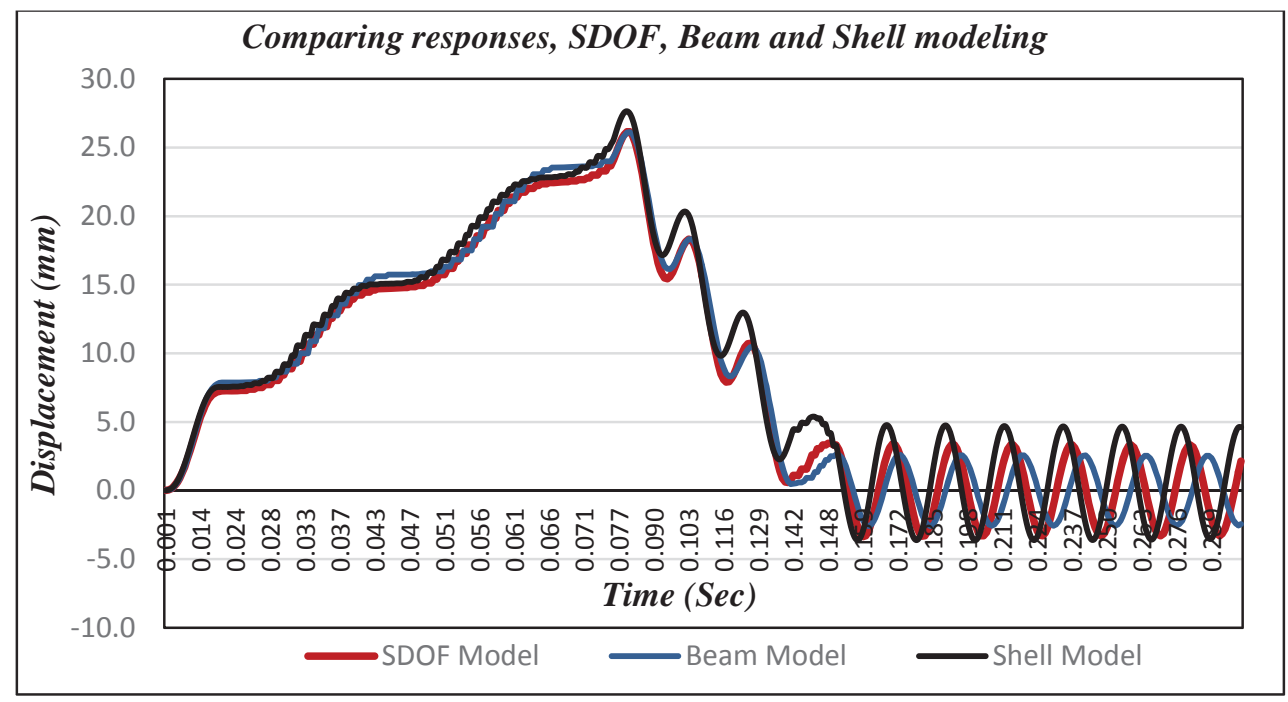

Figure 5. Comparing nonlinear dynamic responses (displacements, $\mathrm{mm}$, for load duration of $2 t_{d}$ ) SDOF, Beam \& Shell models - Model1

\subsection{Modal Analysis and Dynamic Characteristics}

It is crucial to perform modal analysis for understanding the dynamic behaviour or characteristics of profiled barrier blast walls. In the real engineering world, engineers should carry out a model analysis before performing dynamic analyses, to find out the dynamic sensitivity of the structure. In this study, modal analyses were carried out for all randomly developed finite element models, to identify natural period of structure $(T)$. The ratio of $t_{d}$ to $\mathrm{T}$ (i.e. $t_{d} / \mathrm{T}$ ) is a parameter to identify dynamic behaviour of profiled barrier blast wall structure and so this ratio was then investigated as the main parameter to classify whether the response of the wall was classified as impulse, dynamic, quasi static, or static.

The fundamental natural period of a blast wall is the longest natural period at which the structure will respond to any impulse load including explosion. As the overpressure interaction with any object is usually represented as a uniform load, this is the predominant mode of response in most explosion situations. Depending on the value of the load ratio, three or four response regimes are typically defined, denoted respectively as impulse, 
dynamic and quasi-static (or static). Conventionally, the range for fully dynamic response is defined in the IGN guideline (Bowerman et al. 1992) as:

$$
\begin{aligned}
& \frac{t_{d}}{T}<0.4 \quad \text { Impulsive } \\
& 0.4 \leq \frac{t_{d}}{T} \sim 2.0 \quad \text { Dynamic } \\
& \frac{t_{d}}{T}>2.0 \quad \text { Quasi }- \text { Static }
\end{aligned}
$$

This classification seems to be sharp. Czujko (Czujko 2001) also proposed the following classification for the loading regime:

$$
\begin{array}{lr}
\frac{t_{d}}{T} \ll 1.0 & \text { Impulsive } \\
\frac{t_{d}}{T} \sim 1.0 & \text { Dynamic } \\
\frac{t_{d}}{T}>2.0 & \text { Quasi }- \text { Static } \\
\frac{t_{d}}{T}>5.0 & \text { Static }
\end{array}
$$

As can be seen from the given classifications, for the regime in which $t_{d} / \mathrm{T}$ is greater than 2.0, the response of structure is not really dynamic. Norsok (NORSOK 2013) also suggested a classification for the loading regime, but as static loading was not categorized in their classification, it has not been presented here for comparison. Nevertheless, using the Norsok guidelines (NORSOK 2013) and (Det Norske Veritas 2001) for selecting the related regime and recommended $t_{d}$ values, respectively, would result in a similar conclusion for this study. 
Usually, identifying a precise $t_{d}$ is very complicated and challenging as various blast scenarios should be assessed with consideration to all the load uncertainties including magnitude, direction, distance of blast source, type of facilities, congestion and type of ignition. Therefore, using recommended $t_{d}$ values given by the international standards and guidelines is vital and can reduce the risk of considering unsafe values. API and DNV (API 2FB April 2006; Det Norske Veritas. 2001) codes have been chosen to provide the $t_{d}$ values in the assessment for this study. Durations for the positive phase blast loads are expected to vary from $0.2 \mathrm{~s}$ for fairly open compartments to $1 \mathrm{~s}$ for quite closed compartments (API 2FB April 2006; Det Norske Veritas. 2001). It can be seen from these guidelines that the lower bound for $t_{d}$ is limited to $0.1 \mathrm{sec}$ and for most cases $0.2 \mathrm{sec}$ is recommended, unless a more detailed assessment is carried out. It should be noted that the range of values of $t_{d}$, investigated and discussed in this study, has been found suitable for most cases, not only by international codes and standards, but also by industry expertise in blast analyses.

\subsection{Analysis Results}

Before performing any probabilistic analyses, sensitivity studies were carried out to make sure that the model inputs were correctly defined and to have a better understanding of the structural behaviour under general loads and boundary conditions. For each model, 2000 simulation loops were generated for the analyses. The ratio of duration of the loading $\left(t_{d}\right)$ to natural period of structure $(\mathrm{T})$ of the profiled barrier is considered as the characteristic property and investigated.

After performing the probabilistic analyses, it is crucial to review the statistical results to check that the simulation loops are adequate. If the number of simulations is sufficient, the mean value plots for the random output variables converge to a fixed value (i.e., the curve flattens out). As an example, Figure 6 presents the level of satisfaction for the number of loops considered with regards to mean values of $t_{d} / T, t_{d}=0.15 \mathrm{sec}$, for the modal analyses 
associated with Model1 and Model11. It can be seen that the values converge and therefore that the number of samples is valid. The three lines represent the upper, lower and mean values of the output variables.
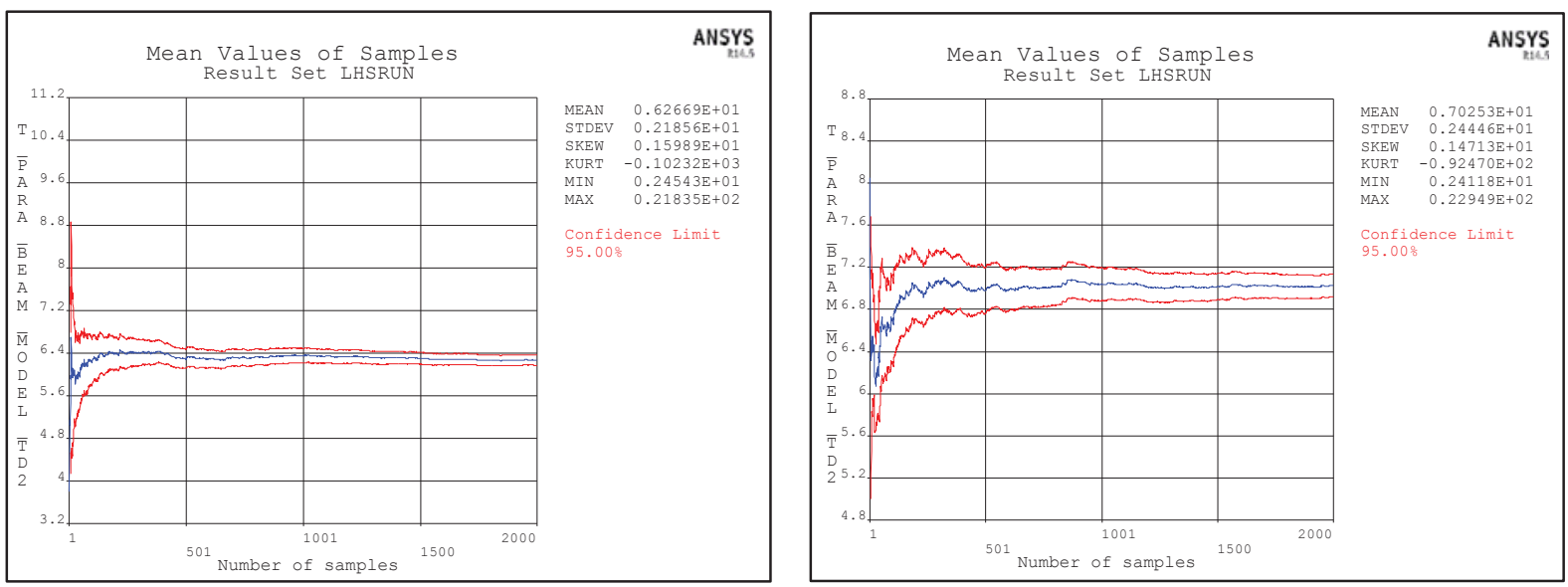

Figure 6. Mean Value of Samples of $t_{d} / T$ at $t_{d}=0.15 \mathrm{sec}$, Model1 (left), Model11 (right)

For the assessment purposes, including structural integrity and risk, it is useful to identify the probability that the maximum response (e.g. Maximum deflection, strain, or stress) remains below a specified limit or value. Furthermore, for design purposes, it is always useful to determine the probability corresponding to the occurrence of the maximum response that satisfies the design requirements. This information can be obtained readily from the cumulative distribution functions (CDFs) of the variables of interest.

As noted previously, based on the international guidelines, one of the criteria associated with section properties for profiled barrier is to limit the angle $(\theta)$ between 45 and 90 degrees which helps to improve the performance of the structures and to reduce the chance of local buckling failure occurring. However, in this study, as the section dimensions, presented in Table 1, are introduced as random variables, there are some cases in which the angle limitation is not satisfied. After generating the models implementing random inputs, the models which satisfy the angle $(\theta)$ limitation have been considered as "valid cases" and 
therefore, the so called "non-valid cases" have generally been removed from the discussions and conclusions of this study.

The probability of having a specific response can be identified using the CDFs plots. Figure 7 shows the cumulative distribution functions associated with $t_{d} / \mathrm{T}$ at $t_{d}=0.15 \mathrm{sec}$, from the modal analyses for the Model1.

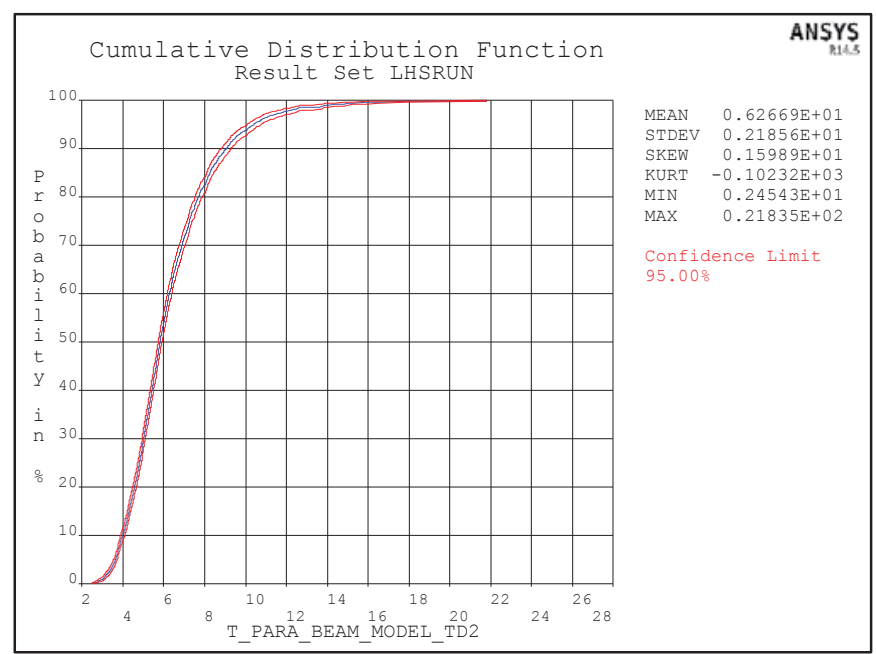

Figure 7. CDF of $t_{d} / T$ at $t_{d}=0.15 s e c$, for the Model1

Table 3 presents the probability or occurrence (\%) of having $t_{d} / \mathrm{T}$ greater than 2.0 for a wide range of $t_{d}$ 's, associated with the valid cases. The total valid cases or runs is 34351 out of 40000 . As can be seen from Table 3, considering a $t_{d}$ of $0.1 \mathrm{sec}$, which is the lower band of the recommended range by API (API 2FB April 2006), and implementing the probabilistic approach presented in this study (with 20 models), it can be concluded that the likelihood of having a dynamic response is $5.8 \%$ (average). In other words, the response of the profiled barrier blast walls is mainly (94.2\% in average), either quasi-static or static. Implementing a $t_{d}$ of 0.2sec, which is recommended by API (API 2FB April 2006) and DNV (Det Norske Veritas. 2001), it can be confirmed that $99.9 \%$ of the structural behaviour is not dynamic. The results from the table also confirm that $99.4 \%$ of the structural response is quasi-static or static when employing a $t_{d}$ of 0.15 sec. 
It should also be noted that these modal analyses are based on the pinned-pinned end condition, with consideration of the weight of Liner and Isolation. Considering a more realistic end or support condition, which is partially fixed (i.e. neither fully pinned nor fully fixed), leads to a smaller value for the natural period ( $T$ ) of the structure and consequently results in a greater value for $t_{d} / \mathrm{T}$ which indicates that the structural responses would move further towards the static or quasi-static domains.

The analysis results also confirm that, because of having a minor probability (e.g., less than $10 \%$ chance) to obtain a dynamic response from an unstiffened profiled barrier under blast loadings, consideration of a dynamic load factor (DLF) of equal 1.1 would be ideal. If not, a proper conservatism should be implemented in the conceptual or preliminary stages of the design and assessment.

The developed probabilistic approach can also be utilised for optimising the design of these structures as well as investigating the local failure modes for profiled barrier blast walls. As part of this research study, further research is being carried out to develop a stochastic finite element framework for profiled barrier stainless steel blast walls. 
Table 3. Occurrence ( $\%$ ) for $t_{d} / T>2.0$ associated with valid cases (Angle $45<\theta<90$ )

\begin{tabular}{|c|c|c|c|c|c|c|c|}
\hline & $t_{d}=0.1$ & $t_{d}=0.15$ & $t_{d}=0.2$ & $t_{d}=0.25$ & $t_{d}=0.3$ & $t_{d}=0.4$ & $\begin{array}{c}\text { Valid cases }(45<\theta<90) \\
\text { out of } 2000\end{array}$ \\
\hline Model1 & 99 & 100 & 100 & 100 & 100 & 100 & 1984 \\
\hline Model2 & 97 & 100 & 100 & 100 & 100 & 100 & 1697 \\
\hline Model3 & 47 & 89 & 99 & 99 & 100 & 100 & 1419 \\
\hline Model4 & 100 & 100 & 100 & 100 & 100 & 100 & 326 \\
\hline Model5 & 99 & 100 & 100 & 100 & 100 & 100 & 316 \\
\hline Model6 & 86 & 99 & 100 & 100 & 100 & 100 & 1696 \\
\hline Model7 & 93 & 100 & 100 & 100 & 100 & 100 & 1889 \\
\hline Model8 & 100 & 100 & 100 & 100 & 100 & 100 & 2000 \\
\hline Model9 & 95 & 100 & 100 & 100 & 100 & 100 & 1937 \\
\hline Model10 & 98 & 100 & 100 & 100 & 100 & 100 & 1709 \\
\hline Model11 & 100 & 100 & 100 & 100 & 100 & 100 & 2000 \\
\hline Model12 & 98 & 100 & 100 & 100 & 100 & 100 & 2000 \\
\hline Model13 & 98 & 100 & 100 & 100 & 100 & 100 & 1998 \\
\hline Model14 & 96 & 100 & 100 & 100 & 100 & 100 & 1999 \\
\hline Model15 & 97 & 100 & 100 & 100 & 100 & 100 & 1990 \\
\hline Model16 & 97 & 100 & 100 & 100 & 100 & 100 & 1971 \\
\hline Model17 & 99 & 100 & 100 & 100 & 100 & 100 & 1984 \\
\hline Model18 & 100 & 100 & 100 & 100 & 100 & 100 & 1968 \\
\hline Model19 & 99 & 100 & 100 & 100 & 100 & 100 & 1864 \\
\hline Model20 & 78 & 99 & 100 & 100 & 100 & 100 & 1604 \\
\hline $\begin{array}{l}\text { Average with } \\
\text { consideration } \\
\text { of valid cases }\end{array}$ & 94.2 & 99.4 & 99.9 & 99.9 & 100.0 & 100.0 & $\begin{array}{c}\text { Total valid runs }= \\
34351\end{array}$ \\
\hline
\end{tabular}

\section{Conclusions}

Initially, an extensive range of programming modules were developed to create a parametric finite element model to analyse and assess profiled barrier blast walls, with consideration of material nonlinearity, and realistic boundary conditions. The option to 
perform various types of analyses including linear, nonlinear, static, dynamic and modal as well as probabilistic assessments were also included. Utilising the programming, validity of the finite element modelling was confirmed. Twenty base-models were then selected or developed from which 34351 valid models were generated probabilistically. For the valid cases, the ratio of load duration to natural period of structure (i.e. $t_{d} / T$ ) was used to identify associated structural response classifications including, impulse, dynamic, quasi-static, and static. The values of $t_{d} / \mathrm{T}$ were examined for a range of $t_{d}$ values, including the ones recommended by international standards and guidelines. The results indicate that when $t_{d}$ is equal to or greater than $0.1 \mathrm{~s}$ (the lower bound and considered to be the worst case scenario), the ratio of $t_{d} / \mathrm{T}$ is mostly greater than 2.0 which implies that the structural response is either static or quasi-static. In fact, it can be concluded that the effects of dynamics are negligible for unstiffened profiled barrier blast walls and structural responses in most cases can be estimated on a quasi-static or static basis. In addition, considering a partially fixed boundary condition, which is more realistic, would decrease the natural period of the structure $(T)$ and consequently increase the ratio of $t_{d} / \mathrm{T}$ and the structural behaviour would be less dynamicsensitive. Using a proper DLF would greatly help design engineers, without involving in complicated advanced nonlinear dynamic analyses at early stage of the assessments. One main direct practical application of the conclusion is to suggest an appropriate DLF of 1.1 to be considered in the early stages of design and assessment. Further study is being carried out to enhance a stochastic numerical approach for profiled barrier stainless steel blast walls. 


\section{Acknowledgments}

This $\mathrm{PhD}$ research is funded by Advanced Structural Analysis and Management Group (ASAMG) Ltd., Aberdeen.

Sriramula's work within the Lloyd's Register Foundation Centre for Safety and Reliability Engineering at the University of Aberdeen is supported by Lloyd's Register Foundation (LRF). LRF, a UK registered charity and sole shareholder of Lloyd's Register Group Ltd, invests in science, engineering and technology for public benefit, worldwide.

\section{References:}

ABS. (2005). "Rules for building and classing steel vessels." .

ABS. (2004). "Guide for buckling and ultimate strength assessment for offshore structures." .

ANSYS. (2012). ANSYS Inc., Documentation. Release 14.0., .

API 2FB. (April 2006). "Recommended Practice for the Design of Offshore Facilities Against Fire and Blast Loading." .

Biggs, J. M. (1964). "Introduction to Structural Dynamics." McGraw-Hill, .

Boh, J. W., Louca, L. A., and Choo, Y. S. (2007). "Finite Element Analysis of Blast Resistant Structures in the Oil and Gas Industry ." ABAQUS Users' Conference, .

Bowerman, H., Graham, W. O., Rumley, J. H., and Tolloczko, J. J. A. (1992). "Interim guidance notes for the design and protection of topside structures against explosion and fire." Steel Construction Institute(Great Britain), .

Brewerton, R., and FABIG TN5. (1999). "Design Guide for Stainless Steel Blast Wall: Technical Note 5, Fire and Blast Information Group (FABIG)." Fire and Blast Information Group (FABIG), .

Choi, S., Grandhi, R. V., and Canfield, R. A. (2006). "Reliability-based Structural Design." Springer, .

Czujko, J., and Paik, J. K. (2015). "A new method for accidental limit states design of thinwalled structures subjected to hydrocarbon explosion loads. " Ships and Offshore Structures " 10(5), 460-469. 
Czujko, Z. (2001). "Design of Offshore Facilities to Resist Gas Explosion Hazard, Engineering Handbook ." CorrOcean ASA, Oslo, Norway, .

Det Norske Veritas. (1992). "Structural Reliability Analysis of Marine Structures: Classification Note No. 30.6." .

Det Norske Veritas. (2001). "Offshore Standard DNV-OS-A101 - Safety Principles and Arrangements." Norway, .

Faruqi, M. A., Grisel, J., Salem, A., and Sai, J. (2010). "A Parametric Study for the Efficient Design of Corrugated Blast Wall Panels Used In Petrochemical Facilities." ARPN Journal of Engineering and Applied Sciences, 5(11), 8996.

Haifu, Q., and Xueguang, L. (2009). "Approach to Blast Wall Structure Computing in Ocean Engineering." Proceedings of the International Conference on Industrial and Information Systems, IIS '09, .

Hancock, G. J., Davids, A. J., Key, P. W., Lau, S. C. W., and Rasmussen, K. J. R. (1990). "Recent developments in the buckling and nonlinear analysis of thin-walled structural members." Thin-Walled Structures, 9(1-4), 309338.

Hedayati, M. H., Sriramula, S., and Neilson, R. D. (2014). "Linear Dynamic Reliability Analysis of Profiled Blast Walls." ASCE-ICVRAM-ISUMA Conference, Liverpool, UK, .

Hedayati, M., Sriramula, S., and Neilson, R. D. (2013). "Non-Linear Dynamic Reliability Analysis of Profiled Blast Walls ." 11th International Conference on Structural Safety and Reliability (ICOSSAR), New York, USA, .

Hedayati, M., Sriramula, S., and Neilson, R. (2015). "Reliability of profiled blast wall structures." In S Kadry \& A El Hami (Eds), Numerical Methods for Reliability and Safety Assessment: Multiscale and Multiphysics Systems. Springer, Switzerland, 387405.

Hedyati, M. H., and Sriramula, S. (2012). "Finite Element Reliability Analysis of Blast Wall." 6th International, ASRANet (Advanced Structural Reliability Analysis Network) Conference, Croydon, London, UK, .

Helton, J. C., and Davis, F. J. (2003). "Latin hypercube sampling and the propagation of uncertainty in analyses of complex systems." Reliab.Eng.Syst.Saf., 81(1), 2369.

Iman, R. L., Helton, J. C., and Campbell, J. E. (1981). "An approach to sensitivity analysis of computer models, Part 1 . Introduction, input variable selection and preliminary variable assessment." Journal of Quality Technology, 13(3), 174-183.

Langdon, G. S., and Schleyer, G. K. (2006). "Deformation and failure of profiled stainless steel blast wall panels. Part III: finite element simulations and overall summary." Int.J.Impact Eng., 32(6), 9881012.

Liang, Y. H., Louca, L. A., and Hobbs, R. E. (2007). "Corrugated panels under dynamic loads." Int.J.Impact Eng., 34(7), 11851201. 
Louca, L. A., and Boh, J. W. (2004). "Analysis and Design of Profiled Blast Walls. Research Report 146." Hse, .

McKay, M. D., Beckman, R. J., and Conover, W. J. (1979). "A comparison of three methods for selecting values of input variables in the analysis of output from a computer code." Technometrics, 21(2), 239-245.

NORSOK. (2013). "Design of Steel Structures." N-004, Rev. 3, .

Olsson, A., Sandberg, G., and Dahlblom, O. (2003). "On Latin hypercube sampling for structural reliability analysis." Struct.Saf., 25(1), 47-68.

Reh, S., Beley, J., Mukherjee, S., and Khor, E. H. (2006). "Probabilistic finite element analysis using ANSYS." Struct.Saf., 28(1-2), 17-43.

Schleyer, G. K., Langdon, G. S., and Jones, N. (2003). "Research Proposal - pulse pressure testing of $1 / 4$ scale blast walls with connections - phase II." HSE Proposal., .

Schleyer, G. K., and Langdon, G. S. (2003). "Pulse pressure testing of 1/4 scale blast wall panels with connections." Prepared by Liverpool University for the Health and Safety Executive, rr124., .

Sohn, J. M., Kim, S. J., Seo, J. K., Kim, B. J., and Paik, J. K. (2016). "Strength assessment of stiffened blast walls in offshore installations under explosions." Ships and Offshore Structures, 11(5), 551-560.

Wijaya, C., and Kim, B. (2011). "FE analysis of unstiffened and stiffened corrugated panels subjected to blast loading." Journal of Mechanical Science and Technology, 25(12), 3159 3164.

Wyss, G. D., and Jorgensen, K. H. (1998). "A user`s guide to LHS: Sandia`s Latin Hypercube Sampling Software, SAND98-0210." Sandia National Lab, . 